\title{
JLab polarized He-3 target
}

\author{
Jie Liu ${ }^{* \dagger}$ \\ University of Virginia \\ E-mail: j15ujevirginia.edu
}

A polarized ${ }^{3} \mathrm{He}$ target was successfully used for fourteen electron scattering experiments at JLab. It is based on the optical pumping of alkali atoms in vapour and the subsequent spin exchange between the polarized alkali atoms and the ${ }^{3} \mathrm{He}$ nuclei. There were significant improvements for this target system during recent years by using the hybrid cell technique (a Rb-K mixture) and newly available high-power, narrow-linewidth diode lasers. The maximum polarization reached over $60 \%$ with electron beam currents up to $15 \mu \mathrm{A}$ on a high density $\left(10^{22} / \mathrm{cm}^{2}\right)$ target, thus establishing a world record for luminosity with a polarized ${ }^{3} \mathrm{He}$ target, $10^{36} / \mathrm{cm}^{2} / \mathrm{s}$. For the 12 $\mathrm{GeV}$ program at JLab, there are seven polarized ${ }^{3} \mathrm{He}$ target experiments approved. Upgrade of the target is underway to meet the requirements of the experiments. The progress is presented.

XVth International Workshop on Polarized Sources, Targets, and Polarimetry September 9 - 13, 2013

Charlottesville, Virginia, USA

\footnotetext{
* Speaker.

${ }^{\dagger}$ On behalf of the JLab polarized ${ }^{3} \mathrm{He}$ target group
} 


\section{Introduction}

Polarized targets are essential for nucleon spin structure study. As a free neutron target cannot be made dense due to its short lifetime ( $885.7 \pm 0.8 \mathrm{~s}$ [1]), the polarized light nuclei targets such as deuteron and ${ }^{3} \mathrm{He}$ are typically used as effective polarized neutron targets. For a ${ }^{3} \mathrm{He}$ nucleus, its ground state is dominated by an $\mathrm{S}$-wave configuration in which the two proton spins are align opposite one another and the neutron accounts for the nuclear spin [2]. Polarized ${ }^{3} \mathrm{He}$ targets have been widely used in neutron electromagnetic and spin structure function study at MIT-Bates, SLAC, DESY, MAMI and JLab.

Two methods are mainly used to polarize ${ }^{3} \mathrm{He}$ nuclei. The first one is based on metastabilityexchange optical pumping (MEOP) [3], where the ${ }^{3} \mathrm{He}$ metastable states are optically pumped directly and subsequently transfer their polarization to ${ }^{3} \mathrm{He}$ ground state nuclei through metastabilityexchange collisions. The second technique is based on spin exchange optical pumping (SEOP) [4], where a vapor of alkali-metal atoms is polarized by laser first and then the polarization is transferred to ${ }^{3} \mathrm{He}$ nuclei in a hyperfine interaction through spin-exchange collisions. The SEOP method was successfully used for fourteen experiments in Hall A at JLab.

There have been significant improvements for the polarized ${ }^{3} \mathrm{He}$ target in recent years. One is the implementation of of a hybrid cell technique (a Rb-K mixture) [5] which greatly improves the spin exchange efficiency [6]. Another advancement is the use of the newly available high-power, narrow-linewidth diode lasers (COMET) instead of the broad-width diode lasers (Coherent) that provide a much higher optical pumping efficiency. By implementing these improvements, the spin up times for the JLab target were shortened from approximately $10 \mathrm{~h}$ to $5 \mathrm{~h}$ [7], and the maximum polarization was increased from $40 \%$ to $60 \%$, with a $15 \mu \mathrm{A}$ electron beam on target [8]. Without beam the polarization reached more than $70 \%$. At $15 \mu \mathrm{A}$, this target $\left(10^{22} / \mathrm{cm}^{2}\right)$ also set a world luminosity record for polarized ${ }^{3} \mathrm{He}, 10^{36} / \mathrm{cm}^{2} / \mathrm{s}$.

\section{Upgrade Plan}

For the $12 \mathrm{GeV}$ program at JLab, there are already seven polarized ${ }^{3} \mathrm{He}$ target related experiments approved. A plan was developed to upgrade the polarized ${ }^{3} \mathrm{He}$ target to meet the requirements of these experiments. The first stage aims to have a $40 \mathrm{~cm}$ long, $10 \mathrm{amg}$ target with $30 \mu \mathrm{A}$ electron beam current and reach an in-beam target polarization of $60 \%$, a factor of 2-3 improvement in the figure of merit. In addition, the precision of polarization measurement will be improved from 5\% to $3 \%$. This target will satisfy the requirements of the Hall A $\mathrm{A}_{1}^{n}$ experiment [9], which is expected to run in 2016.

To reach this goal, R\&D efforts are ongoing to study the performance of the convection cell, a pulsed-NMR system, radiation shielding for high current beam and systematic uncertainties in target polarization measurements [10].

\section{Convection Cell}

A convection type cell was developed and tested at UVa first [11]. The mechanical design of the convection cell system at JLab is shown in Figure 1 and consists of two chambers connected 
by a pair of short tubes. The pumping chamber is a 3.5 inch diameter sphere located inside a 230 ${ }^{\circ} \mathrm{C}$ oven, where ${ }^{3} \mathrm{He}$ nuclei are polarized through spin exchange with a $\mathrm{Rb}-\mathrm{K}$ mixture that is in turn polarized by $795 \mathrm{~nm}$ laser light. The target chamber is a $40 \mathrm{~cm}$ long tube. This design circulates gas between the pumping and target chambers much more rapidly than the previous design, which relied upon diffusion of the gas through a single tube connecting the chambers. Figure 2 shows the setup and typical results for our convective flow measurements at JLab. A small heater on one of the two transfer tubes is used to establish a convective flow of gas between the pumping and target chambers, while a RF pulse from the Free-Induction-Decay (FID) coil is used to perturb the polarization of gas inside the $1.0 \mathrm{inch}$ bulb on the other tube. The polarization dynamics inside the target chamber are then monitored with two sets of pickup coils, using the NMR technique of Adiabatic Fast Passage (AFP). Additional tests to determine the polarization losses due to the AFP measurements themselves have been made with the convection heater on and off, in both the target and pumping chambers. Results are show in Figure 3 and Table 1. The AFP loss with convection on is a little higher, but acceptable.

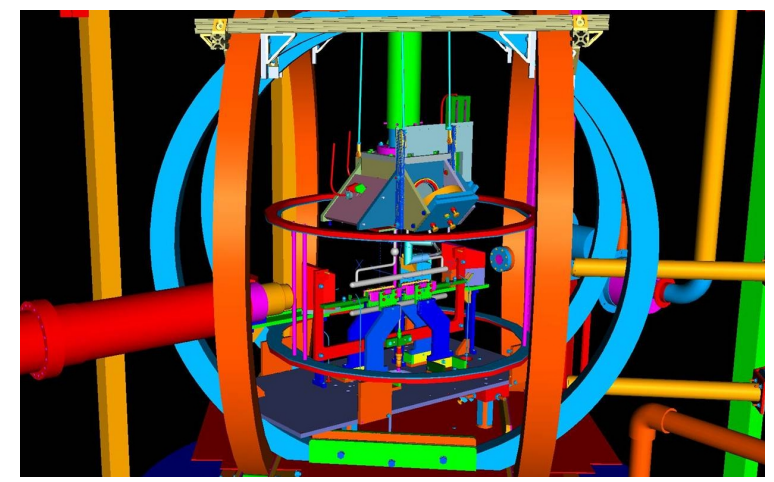

Figure 1: The polarized ${ }^{3} \mathrm{He}$ target mechanical design at JLab.
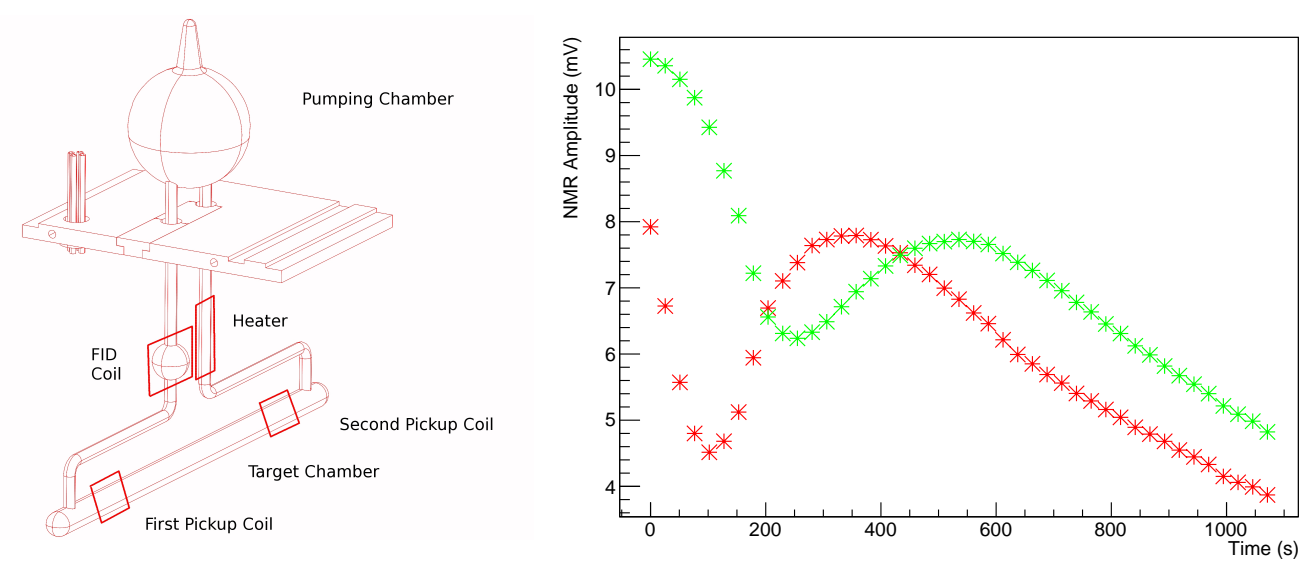

Figure 2: Convection cell set up (left) and NMR signal amplitude versus time from two pickup coils (right). In the right figure, the red and green curves are the NMR amplitudes measured by the first pickup coil and second pickup coil, respectively. The result indicates a gas flow velocity of $5.7 \mathrm{~cm} / \mathrm{min}$ in the target chamber. 

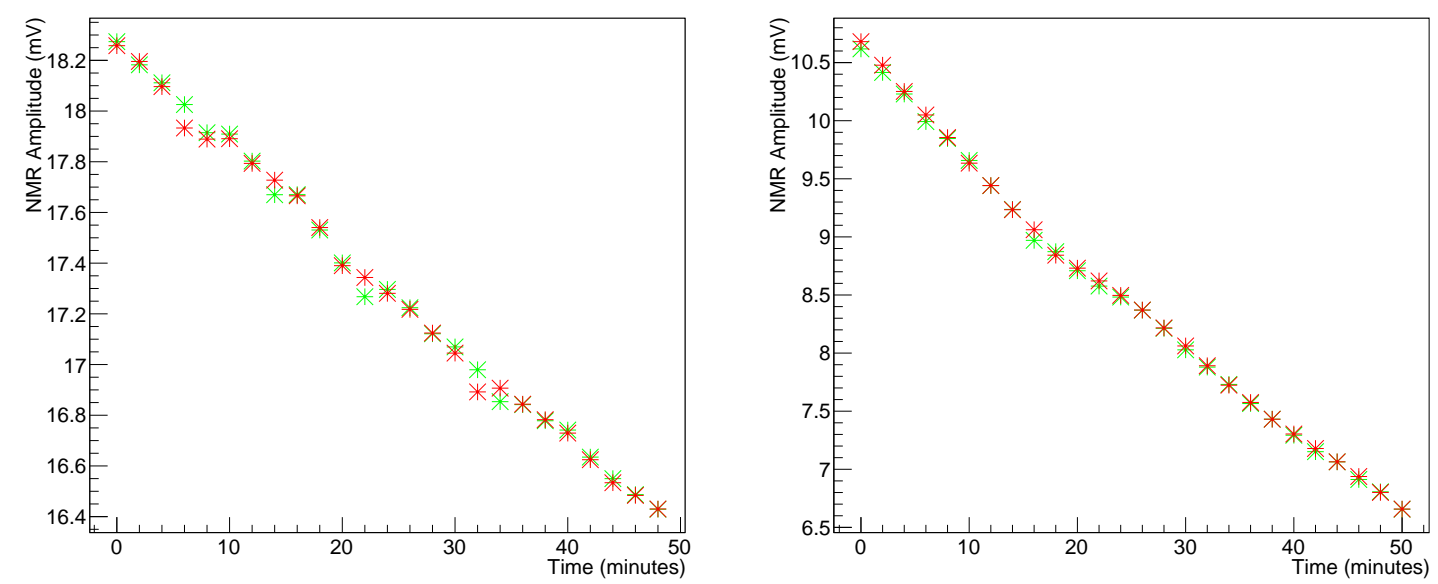

Figure 3: NMR amplitude versus time (25 sweeps * 2 minutes) for target chamber during an AFP loss study. Red and green marks indicate the NMR sweep up and down amplitudes. The result with convection off (on) is shown in left (right) plot.

\begin{tabular}{crr}
\hline \hline AFP Loss Per Sweep & Target Chamber & Pumping Chamber \\
AFP Without Convection & $0.16 \%$ & $0.72 \%$ \\
AFP With Convection & $0.85 \%$ & $0.87 \%$ \\
\hline
\end{tabular}

Table 1: AFP loss with convection on and off for both pumping chamber and target chamber when the laser was off. The results were corrected for polarization loss due to spin relaxation. The uncertainty for AFP loss is about $0.05 \%$.

\section{Pulsed NMR}

Two kinds of polarimetry, AFP-NMR and EPR (Electron Paramagnetic Resonance) were used to measure the polarization of the previous, all-glass ${ }^{3} \mathrm{He}$ target. However, we envision that metal target chambers will be utilized for future experiments with very high luminosity. This will present problems for both AFP and EPR due to attenuation of the RF fields. A pulsed-NMR system was developed at the JLab target lab, which is suitable for measurements on the transfer tube connecting the metal target cells to glass pumping chambers. A RF pulse tuned to the ${ }^{3} \mathrm{He}$ Larmor Frequency, is sent to the FID coil. At resonance in the rotating frame, a spin sees a constant field of $\frac{1}{2} H_{1}$ orthogonal to its spin direction. As a result, the ${ }^{3} \mathrm{He}$ magnetization vector tips away from the holding field through an angle $\theta_{t i p}$ given by

$$
\theta_{\text {tip }}=\frac{1}{2} \gamma H_{1} t_{\text {pulse }},
$$

where the $\gamma$ is the gyro-magnetic ratio and $t_{\text {pulse }}$ is the pulse duration. At the conclusion of the pulse, the spin components orthogonal to the holding field will experience free induction decay with amplitude

$$
\text { Amplitude } \propto M_{z} \sin \left(\theta_{t i p}\right) e^{-t / \tau}
$$


The JLab pulsed-NMR system schematic and typical FID signal are shown in Figure 4. The pulsedNMR results will be cross-calibrated against AFP and EPR measurements made on all glass target systems. Further systematic studies of the pulsed-NMR setup are currently underway.
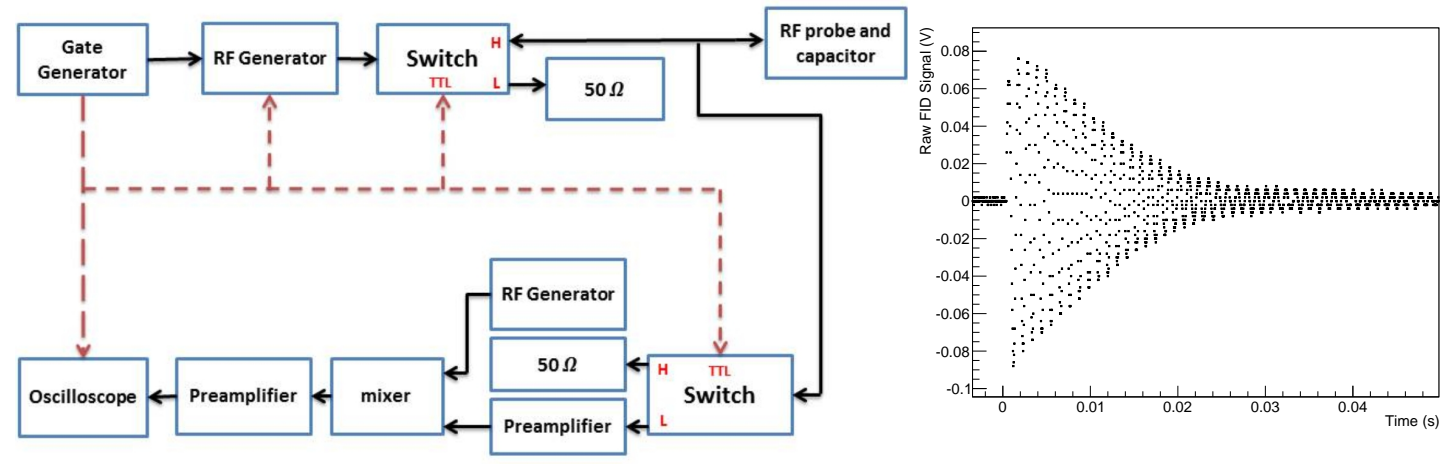

Figure 4: JLab pulsed-NMR system schematic (left) and typical FID signal (right).

\section{Radiation Shielding Study for High Beam Current}

An upgrade of the polarized ${ }^{3} \mathrm{He}$ target is required to be able to handle at least $30 \mu \mathrm{A}$ electron beam for the future experiments. High beam current means more radiation damage to the pumping chamber, which will cause a rupture of the target cell eventually. A radiation shielding study was performed using a detailed Geant 4 simulation, and the results are shown in Figure 5. Doses corresponding to 350 hours of beam time were simulated for both the Transversity [12] and $A_{1}^{n}$ [9] experiments. For a convection cell with a $25 \mathrm{~cm}$ long transfer tube, the radiation damage is less than that of the diffusion cell utilized in the previous Transversity experiment.

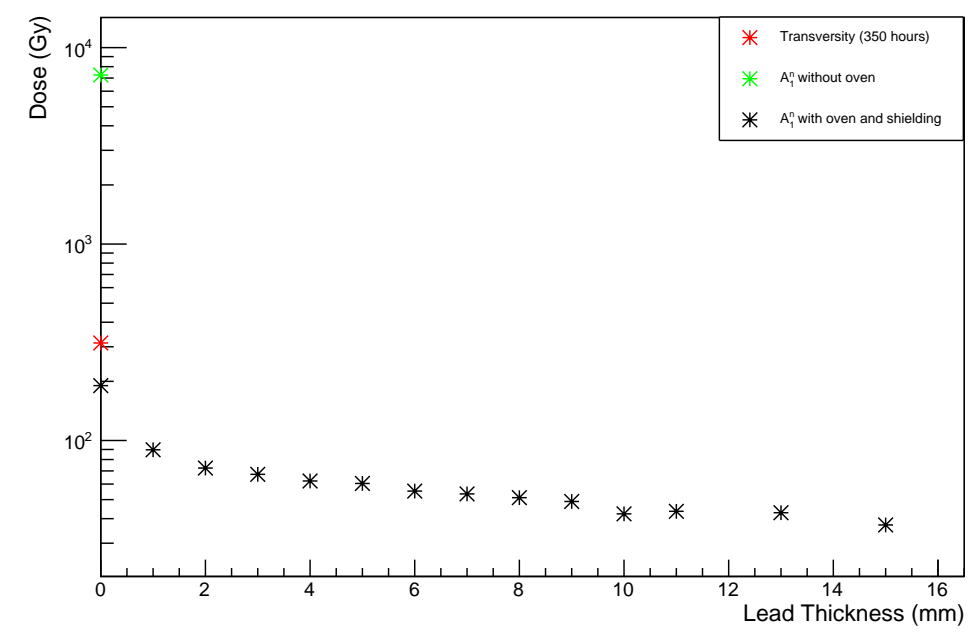

Figure 5: Dose on pumping chamber due to radiation. 


\section{Summary}

The JLab polarized ${ }^{3} \mathrm{He}$ target had a world-record performance during $6 \mathrm{GeV}$ era. R\&D activities are ongoing to upgrade the target for the $12 \mathrm{GeV}$ program. Initial tests of the convection cells and the pulsed-NMR system have been made and proven successful. Full polarization tests and systematic studies are underway. The goal is to have the target system be ready for the $\mathrm{A}_{1}^{n}$ experiment in Hall A by the early 2016.

\section{References}

[1] K. Nakamura et al. (Particle Data Group), J. Phys. G 37, 075021 (2010).

[2] J. L. Friar, B. Gibson, G. Payne, A. Bernstein, and T. Chupp, Phys. Rev. C42, 2310 (1990).

[3] F. Colegrove, L. Schearer, and G.Walters, Phys. Rev. 132, 2561 (1963).

[4] T. E. Chupp, M. E. Wagshul, K. P. Coulter, A. B. McDonald, and W. Happer, Phys. Rev. C36, 2244 (1987).

[5] W. Happer et al., U.S. Patent No. 6,318,092 2001; E. Babcock, et al., Phys. Rev. Lett. 91, 123003 (2003).

[6] A. Ben-Amar Baranga, S. Appelt, M.V. Romalis, C.J. Erickson, A.R. Young, G.D. Cates and W. Happer, Phys. Rev. Lett. 80, 2801 (1998).

[7] Jaideep Singh, Ph.D. thesis, University of Virginia (2010).

[8] Y. Zhang, PhD thesis, Lanzhou University (2011).

[9] JLab E12-06-122, Spokespersons, T. Averett, G. Cates, N. Liyanage, G. Rosner, B. Wojtsekhowski and $\mathrm{X}$. Zheng.

[10] JLab polarized ${ }^{3} \mathrm{He}$ target lab wiki is available at https://hallaweb.jlab.org/wiki/index.php/Target_Lab_Main.

[11] P.A.M. Dolph, J. Singh, T. Averett, A. Kelleher, K.E. Mooney, V. Nelyubin, W.A. Tobias, B. Wojtsekhowski and G.D. Cates, Phys. Rev. C 84, 065201 (2010).

[12] X. Qian et al., Phys. Rev. Lett. 107, 072003 (2011); J. Huang et al., Phys. Rev. Lett. 108, 052001 (2012). 